8 Formen, Ursachen, Entwicklungsstadien und Behandlungsverfahren von Tumorerkrankungen der einzelnen Organe - 1

8.1 Kriterien für Klassifikationen des Tumors, von Risiken, des Allgemeinzustandes, von Operationen und Prozeduren, Therapieerfolgen, Verlaufskontrollen und Leitlinien - 1

8.1.1 Tumorklassifikationen -1

8.1.2 Beurteilung von Tumor-Risiken -5

8.1.3 Beurteilung des Allgemeinzustandes des Patienten - 6

8.1.4 Operationen- und Prozedurenschlüssel (OPS) -7

8.1.5 Beurteilung von Therapieerfolgen - 8

8.1.6 Verlaufskontrolle mit Tumormarkern - 9

8.1.7 Leitlinien für die Diagnose und Therapie - 13

8.2 Bösartige Neubildungen der Brustdrüse (Mamma; C50-C50) - 15

8.2.1 Weibliche Brust -15

8.2.2 Männliche Brust -71

8.3 Bösartige Neubildungen der weiblichen Genitalorgane (ICD10: C51-C58) -78

8.3.1 Eierstock (Ovar) -78

8.3.2 Gebärmutter - 97

8.3.3 Muttermund (Zervix) 123

8.3.4 Scheide/Vagina - $\mathbf{1 4 3}$

8.3.5 Äußeres weibliches Genitale/Vulva — 160

8.4 Bösartige Neubildungen der männlichen Genitalorgane (ICD10: C60-C63) - 175

8.4.1 Hoden -175

8.4.2 Prostata -196

8.4.3 Penis - 217

8.5 Bösartige Neubildungen der Harnorgane (ICD10: C64-C68) - 225

8.5.1 Niere, Nierenbecken und Harnleiter - 225

8.5.2 Harnblase und Harnröhre - 242

8.6 Bösartige Neubildungen der Epithelien des Kopfes und Halses (CD10: C00-C14) - 262

8.6.1 Lippen, Mundhöhle, Pharynx, Nasenhöhle, Larynx, Speicheldrüsen - 262

8.6.2 Zahnanlagen - 283

8.6.3 Speiseröhre/Ösophagus - 287

8.7 Bösartige Neubildungen der Verdauungsorgane (ICD10: C15-C26) - 304

8.7.1 Magen -304

8.7.2 Dünndarm - 321

8.7.3 Dickdarm, Enddarm - $\mathbf{3 2 7}$

8.7.4 Anus - 357

8.7.5 Leber -366 
8.7.6 Gallengänge/Gallenwege und Gallenblase - 379

8.7.7 Bauchspeicheldrüse - $\mathbf{3 9 3}$

8.8 Bösartige Neubildungen der Lunge und Mesotheliome des Pleuraendothels (ICD10: C30-C39) - 405

8.8.1 Klassifikation der malignen Lungentumore - $\mathbf{4 0 5}$

8.8.2 Kleinzellige (SCLC) und Nicht-Kleinzellige (NSCLC) Lungen (Bronchial-)Karzinome - $\mathbf{4 0 8}$

8.8.2 Mesotheliome (Lunge/Pleura) - 435

8.9 Bösartige Neubildungen des Knochens und des Gelenkknorpels (ICD10: C40-C41) — 443

8.10 Bösartige Neubildungen der Haut (ICD10: C43-C44) - 456

8.10.1 Melanome - $\mathbf{4 5 6}$

8.10.2 Nicht-melanozytärer Hautkrebs (Aktinische Keratosen, Plattenepithelkarzinome, Basalzellkarzinome) -477

8.10.3 Merkel-Zell-Karzinome - $\mathbf{4 9 6}$

8.10.4 Übrige maligne Tumoren der Haut - $\mathbf{5 0 5}$

8.11 Bösartige Neubildungen des Weichteilgewebes (ICD10: C45-C49) — 506

8.11.1 Sarkome der Muskel-, Fett-, Bindegewebs- und Gefäßzellen und Tumoren des peripheren neuralen und perineuralen Gewebes - $\mathbf{5 0 7}$

8.11.2 Gastrointestinale Stromatumoren - $\mathbf{5 2 7}$

8.11.3 Ewing-Sarkome - $\mathbf{5 4 2}$

8.11.4 Uterussarkome $-\mathbf{5 4 3}$

8.11.5 Kaposi-Sarkome $-\mathbf{5 5 4}$

8.11.6 Tumoren des Peritonealepithels (Peritoneal-Mesotheliome) $\mathbf{- 5 6 4}$

8.12 Bösartige Neubildungen des Auges, des Gehirns und sonstiger Teile des Nervensystems (ICD10: C69-C72) - 565

8.12.1 Tumoren des ZNS/Zentralen Nervensystems - $\mathbf{5 6 5}$

8.12.2 Neuroepitheliale Tumoren (Gliome, Ependymome, neurale Tumore, Plexus-und Pinealis-Tumore, embryonale Tumore) $-\mathbf{5 7 3}$

8.12.3 Nicht-neuroepitheliale Tumoren - $\mathbf{5 8 6}$

8.12.4 Metastasen extrazerebraler Tumoren - 600

8.12.5 Tumoren des PNS/peripheren Nervensystems - 604

8.12.6 Periphere neurale Neuroendokrine Tumoren/periphere neurale NET - 610

8.13 Periphere epitheliale neuroendokrine Tumoren (NET) - 629

8.13.1 Übersicht (Vorkommen, Klassifikation, Diagnostik, Risikofaktoren und Prognose) - 629

8.13.2 Neuroendokrine Tumoren der Lunge - 637

8.13.3 Neuroendokrine Tumore des Gastrointestinaltraktes — 641

8.13.4 Pankreatische NET/Tumoren der Pankreasinseln — 666

8.13.5 Schilddrüsenkarzinome - 672

8.13.6 Nebenschilddrüsenkarzinom - 682

8.13.7 Nebennierenrindenkarzinom — 687

8.14 Tumoren mit unbekannten Primärtumoren/CUP/Cancer of unknown Primaries - 693 
8.15 Bösartige Neubildungen des lymphatischen, blutbildenden Gewebes (ICD10:

$$
\text { (81-C96) - } 698
$$

8.15.1 Übersicht -698

8.15.2 MDS/Myelo-Dysplastische Syndrome — 703

8.15.3 Akute Leukämien - 713

8.15.4 Chronische Lymphozyten-Leukämien — 747

8.15.5 Myeloproliferative Neoplasien (MPN) -784

8.15.6 Myelome/Plasmazelltumore - $\mathbf{8 5 4}$

8.15.7 HL/Hodgkin-Lymphome - $\mathbf{8 6 9}$

8.15.8 NHL/Non-Hodgkin-Lymphome - $\mathbf{8 8 1}$

8.15.9 B-Zell-Lymphome - $\mathbf{8 9 3}$

8.15.10 PTCL/Periphere T-Zell-Lymphome - $\mathbf{9 4 0}$

8.15.11 PTLD/Posttransplantations-lymphoproliferative Erkrankung - 948

8.15.12 Echte histiozytäre Lymphome - 949

8.15.13 Primäre Effusions-Lymphome — $\mathbf{9 4 9}$

8.15.14 Lymphome der Haut — 949

8.15.15 PCNSL/Primäre ZNS-Lymphome — 959

8.15.16 Primäre Lymphome des Gastrointestinaltraktes — 962

Schlussbemerkung und Haftungsausschluss - 969

Sachregister -971

Über den Autor — 1003

Die Kapitel 1-7 finden Sie in Onkologie - die Tumorerkrankungen des Menschen,

Band 1: Entstehung, Wachstum, Diagnostik- und Therapiemöglichkeiten,

ISBN 978-3-11-064787-7.

\section{Inhalt Band 1}

1 Der Begriff Tumor - 1

2 Häufigkeiten von Tumorerkrankungen - 19

3 Regelkreise der Wachstumskontrolle normaler Zellen - 47

$4 \quad$ Krebsentstehung (Karzinogenese) - 255

$5 \quad$ Wachstum von Tumoren - 427

$6 \quad$ Kontrolle des Tumorwachstums durch das Immunsystem -485

7 Grundzüge der Tumortherapie -649 
$X$ Inhalt

Schlussbemerkung und Haftungsausschluss -847

Sachregister - 849

Über den Autor — 883 